\title{
NUMERICAL APPROXIMATION OF SELF-CONSISTENT VLASOV MODELS FOR LOW-FREQUENCY ELECTROMAGNETIC PHENOMENA
}

\author{
Nicolas BESSE*, NORbert J. MAUSER **, ERIC SONNENDRÜCKER *** \\ * Laboratoire de Physique des Milieux Ionisés et Applications \& Institut de Mathématiques Elie Cartan \\ Université Henri Poincaré Nancy-I \\ BP 23954506 Vandoeuvre-lès-Nancy Cedex, France \\ e-mail: besse@iecn.u-nancy.fr;Nicolas.Besse@lpmi.uhp-nancy.fr \\ ** Wolfgang Pauli Institute c/o Faculty of Mathematics, University of Vienna \\ Nordbergstrasse 15, A-1090 Wien, Austria \\ e-mail: norbert.mauser@univie.ac.at \\ *** Institut de Recherche Mathématique Avancée, UMR 7501 CNRS/ULP \\ 7 rue René Descartes, 67084 Strasbourg Cedex, France \\ e-mail: sonnen@math.u-strasbg.fr
}

\begin{abstract}
We present a new numerical method to solve the Vlasov-Darwin and Vlasov-Poisswell systems which are approximations of the Vlasov-Maxwell equation in the asymptotic limit of the infinite speed of light. These systems model low-frequency electromagnetic phenomena in plasmas, and thus "light waves" are somewhat supressed, which in turn allows the numerical discretization to dispense with the Courant-Friedrichs-Lewy condition on the time step. We construct a numerical scheme based on semi-Lagrangian methods and time splitting techniques. We develop a four-dimensional phase space algorithm for the distribution function while the electromagnetic field is solved on a two-dimensional Cartesian grid. Finally, we present two nontrivial test cases: (a) the wave Landau damping and (b) the electromagnetic beam-plasma instability. For these cases our numerical scheme works very well and is in agreement with analytic kinetic theory.
\end{abstract}

Keywords: Vlasov-Darwin model, Vlasov-Poisswell model, semi-Lagrangian methods, low-frequency electromagnetic phenomena

\section{Introduction}

The physics of plasma and charged particle beams involves the interaction of charged particles through their self-induced electromagnetic fields. A description of these phenomena at the microscopic level through the law of dynamics is not usable for numerical simulations due to the huge amount of particles involved. Therefore, approximate models have to be used. Such models are generally of two kinds: kinetic models, which allow for nonequilibrium velocity distributions, and fluid models which are macroscopic models, valid when the particles are at a thermodynamical equilibrium.

Kinetic models, such as the Vlasov equation, are necessary to account for a wide range of physical phenomena. The Vlasov equation needs to be coupled nonlinearly to a model describing the evolution of the self-induced elec- tromagnetic fields. Such a model is given by Maxwell's equations. However, in some cases, it is not necessary to solve the numerically relatively costly Maxwell equations, as approximate models which still describe the relevant physics can be solved more efficiently.

One of these well-known approximate models which has been used in accelerator physics as well as in plasma physics is the Darwin model (Borodachev, 2005; Gibbons and Hewett, 1995; Gibbons and Hewett, 1997; Lee et al., 2001; Schmitz and Grauer, 2006; Sonnendrucker et al., 1995). It is obtained from Maxwell's equations by neglecting the solenoidal, i.e., divergence-free part of the displacement current in Ampère's law. This procedure transforms the hyperbolic Maxwell equations into a set of three elliptic equations and thus adds some regularization features in the model as well as removes a time-step con- 
straint for the stability of explicit numerical schemes.

In contrast to the "quasi-static model" (Maxwell equations where all times derivates are supressed; in other words, it is the model formed by the electrostic and magnetostatic equations), the Darwin model is consistent with the charge conservation or continuity equation that results from the Vlasov equation. This is also the case of an even simpler model, called "Poisswell", which has been recently introduced by Masmoudi and Mauser (2001) as a "first-order" relativistic correction to the Poisson equation, for the sake of a consistent coupling to the Pauli equation as a first-order relativistic correction of the Schrödinger equation.

The numerical solution of the Vlasov equation is mostly performed with Particle-In-Cell (PIC) methods, which have the advantage of giving qualitatively accurate solutions at a relatively low cost. Vlasov-Darwin PIC methods were developed in (Gibbons and Hewett, 1995; Gibbons and Hewett, 1997; Sonnendrucker et al., 1995). However, when higher accuracy is required, it is often useful to solve the Vlasov equation on a phase space grid. Such a method for the Vlasov-Darwin model has been introduced very recently (Schmitz and Grauer, 2006). It is based on a flux conservative scheme (Fijalkow, 1999; Filbet et al., 2000; Sabatier et al., 1990). Our aim is to present an alternative numerical method for the VlasovDarwin and also Vlasov-Poisswell models which is based on a semi-Lagrangian method for the Vlasov equation. Finally, we mention some analytic works on the VlasovDarwin model. The existence of a global-in-time weak solution is proven in (Benachour et al., 2003) in the case of small initial data. In (Pallard, 2006), the author investigates global-in-time weak solutions for general initial data and proves the existence of a unique local-intime strong solution for smooth data. In (Degond and Raviart, 1992; Raviart and Sonnendrücker, 1996), the authors make an asymptotic study of the Maxwell equations and show that the Darwin model approximates the Maxwell system up to the second order with respect to the small parameter $v / c$. Finally, in (Bauer and Kunze, 2005), there is a proof of the convergence of the Vlasov-Maxwell system towards the Vlasov-Darwin system in the Newtonian limit $c \rightarrow \infty$, with a convergence rate of $\mathcal{O}\left(c^{-3}\right)$.

This paper is organized as follows: First, we introduce and derive the Darwin and Poisswell models from Maxwell's equations. Then, we present the numerical method that we use for the solution of the Vlasov-Darwin and Poisswell equations. It is based on a time split semiLagrangian method for the Vlasov equation and classical finite difference or finite element methods for the field equations. Finally, we validate our scheme by presenting numerical results for the Landau damping and the electromagnetic beam plasma instability test cases.

\section{Vlasov-Darwin and Vlasov-Poisswell Models}

Let $L$ be the characteristic length of the problem, $\tau$ its characteristic time and $c$ the speed of light. Then $\varepsilon=$ $L / \tau c$ is a dimensionless parameter and the corresponding dimensionless relativistic Vlasov equation is

$$
\partial_{t} f+\boldsymbol{v}(\boldsymbol{\xi}) \cdot \nabla_{\boldsymbol{x}} f+\boldsymbol{F}(t, \boldsymbol{x}, \boldsymbol{\xi}) \cdot \nabla_{\boldsymbol{\xi}} f=0,
$$

where $f(t, \boldsymbol{x}, \boldsymbol{\xi})$ represents the distribution function of one species of particles (ions, electrons), depending on time $t$, position $\boldsymbol{x}$, and momentum $\boldsymbol{\xi}$. The force field $\boldsymbol{F}(t, \boldsymbol{x}, \boldsymbol{\xi})$ is given here by the Lorentz force

$$
\boldsymbol{F}(t, \boldsymbol{x}, \boldsymbol{\xi})=q(\boldsymbol{E}+\varepsilon \boldsymbol{v}(\boldsymbol{\xi}) \times \boldsymbol{B}),
$$

where $q= \pm 1$ is the sign of the charge. The relativistic velocity of particles $\boldsymbol{v}(\boldsymbol{\xi})$ is given by

$$
\boldsymbol{v}(\boldsymbol{\xi})=\frac{\boldsymbol{\xi}}{\gamma(\boldsymbol{\xi})}=\frac{\boldsymbol{\xi}}{\sqrt{1+\varepsilon^{2}|\boldsymbol{\xi}|^{2}}} .
$$

From the distribution function $f$, we compute the charge and the current densities

$$
\begin{aligned}
& \left.\rho(t, \boldsymbol{x})=q \int_{\mathbb{R}^{3}} f(t, \boldsymbol{x}, \boldsymbol{\xi})\right) \mathrm{d} \boldsymbol{\xi}, \\
& \boldsymbol{j}(t, \boldsymbol{x})=q \int_{\mathbb{R}^{3}} \boldsymbol{v}(\boldsymbol{\xi}) f(t, \boldsymbol{x}, \boldsymbol{\xi}) \mathrm{d} \boldsymbol{\xi},
\end{aligned}
$$

respectively. The electromagnetic field $(\boldsymbol{E}, \boldsymbol{B})$ has to solve some field equations of which the charge density $\rho$ and current density $\boldsymbol{j}$ are the source terms. Moreover, the field equations for $(\boldsymbol{E}, \boldsymbol{B})$ must satisfy the charge conservation equation

$$
\partial_{t} \rho+\nabla \cdot \boldsymbol{j}=0
$$

which is obtained by integrating the Vlasov equation (1) with respect to $\boldsymbol{\xi}$. This compatibility condition is necessary to couple the Vlasov equation to electromagnetic field equations.

Now we present two models of the electromagnetic field whose common feature is the fact that they only take into account the "low-frequency part" of the electromagnetic field.

2.1. Darwin Model. The Darwin approximation of Maxwell's equations is introduced in order to remove the time scale of light waves. This model eliminates high frequency electromagnetic waves, but keeps an important part of the physics in the low-frequency range. The electric field $\boldsymbol{E}$ is decomposed into two parts, i.e., an irrotational part $\boldsymbol{E}_{\text {irr }}$, which is curl free, and a solenoidal part $\boldsymbol{E}_{\mathrm{sol}}$, which is divergence free, i.e., $\boldsymbol{E}=\boldsymbol{E}_{\mathrm{irr}}+\boldsymbol{E}_{\mathrm{sol}}$, 
where $\nabla \times \boldsymbol{E}_{\text {irr }}=0$ and $\nabla \cdot \boldsymbol{E}_{\mathrm{sol}}=0$. Furthermore, as $\nabla \times \boldsymbol{E}_{\text {irr }}=0$, we can write $\boldsymbol{E}_{\text {irr }}=-\nabla \phi$. The Darwin approximation consists in dropping the solenoidal part of the displacement current from Ampère's law written here in an adimensional form:

$$
\frac{1}{\varepsilon} \nabla \times \boldsymbol{B}=\boldsymbol{j}+\partial_{t} \boldsymbol{E}_{\mathrm{irr}}+\partial_{t} \boldsymbol{H}_{\mathrm{sol}}
$$

where $\varepsilon=L / \tau c$ with $L$ being the characteristic length of the problem, $\tau$ its characteristic time, e.g., the frequency in which we are interested, and $c$ the velocity of light. By taking the curl of this equation, since $\nabla \cdot \boldsymbol{B}=0$, we obtain

$$
-\Delta \boldsymbol{B}=\varepsilon \nabla \times \boldsymbol{j} .
$$

By dropping only the solenoidal part of the displacement current, charge conservation is retained in the Darwin model.

Taking the curl of Faraday's law and using the time derivate of Ampère's law, we obtain an elliptic equation for $\boldsymbol{E}_{\mathrm{sol}}$ :

$$
\begin{aligned}
-\Delta \boldsymbol{E}_{\mathrm{sol}} & =-\varepsilon^{2}\left(\partial_{t} \boldsymbol{j}+\partial_{t}^{2} \boldsymbol{E}_{\mathrm{irr}}\right) \\
& =-\varepsilon^{2}\left(\partial_{t} \boldsymbol{j}-\partial_{t} \boldsymbol{j}_{\mathrm{irr}}\right) \\
& =-\varepsilon^{2} \partial_{t} \boldsymbol{j}_{\mathrm{sol}},
\end{aligned}
$$

where we used $\nabla \cdot \boldsymbol{E}_{\mathrm{sol}}=0$ and set

$$
\boldsymbol{j}=\boldsymbol{j}_{\text {irr }}+\boldsymbol{j}_{\mathrm{sol}}, \quad \partial_{t} \boldsymbol{E}_{\text {irr }}+\boldsymbol{j}_{\text {irr }}=0 .
$$

Finally, the Poisson law, $\nabla \cdot \boldsymbol{E}_{\mathrm{irr}}=\rho$, gives Poisson's equation for the electrostatic potential

$$
-\Delta \phi=\rho .
$$

Instead of Maxwell's equations, which are hyperbolic, we now have three elliptic equations. Thus the (CFL) condition on stability no longer constrains the time step.

Using a formal Hilbert expansion of $\boldsymbol{E}$ and $\boldsymbol{B}$ with respect to $\varepsilon$,

$$
\begin{aligned}
& \boldsymbol{E}=\boldsymbol{E}_{0}+\varepsilon \boldsymbol{E}_{1}+\cdots, \\
& \boldsymbol{B}=\boldsymbol{B}_{0}+\varepsilon \boldsymbol{B}_{1}+\cdots,
\end{aligned}
$$

it was shown (Degond and Raviart, 1992; Raviart and Sonnendrücker, 1996) that the "quasistatic approximation", which consists in neglecting all time derivatives in Maxwell's equation, is a simple first-order approximation of Maxwell equations, i.e., $\boldsymbol{E}_{0}$ and $\boldsymbol{B}_{0}$ are the same but $\boldsymbol{E}_{1}$ and $\boldsymbol{B}_{1}$ differ. Such a formal asymptotic expansion shows that the Darwin model coincides with Maxwell's equations up to the second order. Note that the "quasistatic model" which adds the magnetostatic equation for the magnetic field to the Poisson equation has the drawback that its coupling to the Vlasov equation is inconsistent with charge conservation.
Therefore, the Darwin approximation is justified for small $\varepsilon$, e.g., in the nonrelativistic regime. In some situations, especially in magnetized plasma, it is essential to keep the first-order term, which will support electromagnetic phenomena. These includes Alfven waves, ion micro-instabilities, filamentation instabilities, electromagnetic beam-plasma instabilities (Califano et al., 1998) and tearing instabilities (Taguchi et al., 2001).

2.2. Poisswell Model. In the previous section we have seen that the Darwin field-model is an approximation of the second order in $\varepsilon$ of Maxwell's equations that yields a good model when coupled to the Vlasov equation, whereas the "quasistatic model" is not a good first-order model given by a slightly different formulation that we call the Poisswell field-model. This model was introduced in quantum mechanics (Masmoudi and Mauser, 2001) to add the self-consistent electromagnetic field of the or$\operatorname{der} \varepsilon$ to the Pauli wave-function equation which is also an approximation of the order $\varepsilon$ of the Dirac equation. Let us first recall Maxwell's equations with the scaling $\varepsilon=L / \tau c$ :

$$
\begin{aligned}
& \nabla \times \boldsymbol{B}=\varepsilon\left(\boldsymbol{j}+\partial_{t} \boldsymbol{E}\right), \quad \nabla \cdot \boldsymbol{E}=\rho, \\
& \varepsilon \partial_{t} \boldsymbol{B}+\nabla \times \boldsymbol{E}=0, \quad \nabla \cdot \boldsymbol{B}=0 .
\end{aligned}
$$

If we introduce the potentials $\phi$ and $\boldsymbol{A}$, the electromagnetic field is defined as

$$
\boldsymbol{E}=-\nabla \phi-\varepsilon \partial_{t} \boldsymbol{A}, \quad \boldsymbol{B}=\nabla \times \boldsymbol{A} .
$$

In order for the field to be well determined by the potentials, we have to add a gauge. We choose the Lorentz gauge which, in our scaling, is

$$
\varepsilon \partial_{t} \phi+\nabla \cdot \boldsymbol{A}=0 .
$$

We will see that the Lorentz gauge allows us to recover the charge conservation equation. If we plug the expansion (10) and the following Hilbert expansions for $\phi$ and $\boldsymbol{A}$ :

$$
\boldsymbol{A}=\boldsymbol{A}_{0}+\varepsilon \boldsymbol{A}_{1}+\cdots, \quad \phi=\phi_{0}+\varepsilon \phi_{1}+\cdots
$$

into (11), (12) and (13), (14), and collect terms of equal powers in $\varepsilon$, we get

$\mathcal{O}(1)$ :

$$
\begin{aligned}
& \nabla \times \boldsymbol{E}_{0}=0, \quad \nabla \cdot \boldsymbol{E}_{0}=\rho, \\
& \nabla \times \boldsymbol{B}_{0}=0, \quad \nabla \cdot \boldsymbol{B}_{0}=0 \text { hence } \boldsymbol{B}_{0}=0, \\
& \boldsymbol{E}_{0}=-\nabla \phi_{0},
\end{aligned}
$$

$\mathcal{O}(\varepsilon)$

$$
\begin{aligned}
& \nabla \times \boldsymbol{B}_{1}=\partial_{t} \boldsymbol{E}_{0}+\boldsymbol{j}, \quad \nabla \cdot \boldsymbol{B}_{1}=0, \\
& \nabla \times \boldsymbol{E}_{1}=0, \quad \nabla \cdot \boldsymbol{E}_{1}=0 \text { hence } \boldsymbol{E}_{1}=0, \\
& \partial_{t} \phi_{0}+\nabla \cdot \boldsymbol{A}_{1}=0 .
\end{aligned}
$$


We have $\boldsymbol{A}=\varepsilon \boldsymbol{A}_{1}+\mathcal{O}\left(\varepsilon^{2}\right)$ since $\boldsymbol{A}_{0}=0$, and $\phi=$ $\phi_{0}+\mathcal{O}\left(\varepsilon^{2}\right)$ since $\phi_{1}=0$ from (17) and (20). From (16) and (18) on the one hand and from (19), (21) and (18) on the other hand, we get the following "Poisswell system" as the approximation of the Maxwell equation (11)-(12) at $\operatorname{order} \varepsilon$ :

$$
-\Delta \phi=\rho, \quad-\Delta \boldsymbol{A}=\varepsilon \boldsymbol{j} .
$$

Since $\boldsymbol{B}_{0}$ and $\boldsymbol{A}_{0}$ vanish, the magnetic field/potential $\boldsymbol{B}$, $\boldsymbol{A}$ is relativistic at order $\varepsilon$ and, consequently, the scaled Lorentz force is relativistic at order $\varepsilon^{2}$. If we differentiate (22) with respect to time, multiply it by $\varepsilon$ and add the result to the divergence of (9), we get

$$
-\varepsilon\left(\partial_{t} \rho+\nabla \cdot \boldsymbol{j}\right)=\Delta\left(\varepsilon \partial_{t} \phi+\nabla \cdot \boldsymbol{A}\right)=0 .
$$

Thus we recover the equation of charge conservation and the "Poisswell model" is compatible with the Vlasov equation, which is not the case for the "quasistatic equation." Finally, if we take the curl of (22), we get the equation for the magnetic field

$$
-\Delta \boldsymbol{B}=\varepsilon \nabla \times \boldsymbol{j},
$$

which is the same as for the Darwin model (6). If we differentiate (22) with respect to time, we get an equation for $-\varepsilon \partial_{t} A$, the second part of the electric field (13) of the Poisswell model

$$
-\Delta\left(-\varepsilon \partial_{t} \boldsymbol{A}\right)=-\varepsilon^{2} \partial_{t} \boldsymbol{j} .
$$

Note the difference between this result and Eqn. (7) for the solenoidal part of the electric field of the Darwin model. In fact, these two electrical fields are different. One is divergence free $\left(\boldsymbol{E}_{\mathrm{sol}}\right)$ and the other is not $\left(-\varepsilon \partial_{t} \boldsymbol{A}\right)$. In fact, the gauge for the potentials associated to the Darwin electromagnetic field is the Coulomb gauge $(\nabla \cdot \boldsymbol{A}=0$ ), whereas for the Poisswell model it is the Lorentz gauge. It is interesting to observe that this choice of the gauge is the good one-intuitively, one might think that the Coulomb gauge, which is the nonrelatvistic limit of the Lorentz gauge, fits better to a "first-order" nonrelativistic model.

\section{Numerical Approximation}

In this section we recall the semi-Lagrangian method with a time splitting strategy (Strang splitting is often a natural choice). Next we focus on particular problems posed by the Darwin and Poisswell models. Finally, we state the complete algorithm.

3.1. Semi-Lagrangian Method with Time Splitting. The integration of the Vlasov equation using a semiLagrangian method and time splitting à la Strang was widely used (Bégué et al., 1999; Besse, 2004; Besse and Mehrenberger, 2006; Besse and Sonnendrücker, 2003; Cheng and Knorr, 1976). Roughly speaking, it consists in splitting the full transport operator into two transport operators-the first in the physical space, the second in the velocity or momentum space-and solving them successively in a right order to get a high-order approximation in time of the complete transport operator. Let $\mathcal{M}_{h}$ be a discretization of the phase space and $f_{h}^{n}$ an approximation of $f$ at time $t^{n}$ on $\mathcal{M}_{h}$. The general algorithm to compute $f_{h}^{n+1}$ consists of the following three steps:

Step 1. Half time advection in physical space. This step consists in solving the equation

$$
\begin{aligned}
\partial_{t} f+\boldsymbol{v}(\boldsymbol{\xi}) \cdot \partial_{\boldsymbol{x}} f & =0, \quad t \in\left[t^{n}, t^{n+1 / 2}\right], \\
f\left(t^{n}\right) & =f_{h}^{n} .
\end{aligned}
$$

In order to solve (25), we integrate its associated characteristic curve equation

$$
\frac{\mathrm{d} \boldsymbol{X}}{\mathrm{d} t}(t)=\boldsymbol{v}(\boldsymbol{\Xi}(t))
$$

on the time interval $\left[t^{n}, t^{n+1 / 2}\right]$. Then we get

$$
\begin{aligned}
\boldsymbol{X}^{n}-\boldsymbol{X}^{n+1 / 2} & =\int_{t^{n+1 / 2}}^{t^{n}} \boldsymbol{v}(\boldsymbol{\Xi}(t)) \mathrm{d} t \\
& =\int_{t^{n+1 / 2}}^{t^{n}} \boldsymbol{v}\left(\boldsymbol{\Xi}\left(t^{n}\right)\right) \mathrm{d} t+\mathcal{O}\left(\Delta t^{2}\right) \\
& \simeq-\boldsymbol{v}\left(\boldsymbol{\Xi}^{n}\right) \Delta t / 2,
\end{aligned}
$$

where $\left(\boldsymbol{X}^{n+1 / 2}, \boldsymbol{\Xi}^{n}\right) \in \mathcal{M}_{h}$, and $\boldsymbol{X}^{n}$ is the origin of the characteristic curve we look for. Therefore, the new distribution function is such that

$$
\begin{aligned}
f_{h}^{\star}(\boldsymbol{x}, \boldsymbol{\xi}) & :=\tilde{f}_{h}^{n+1 / 2}\left(\boldsymbol{X}^{n+1 / 2}, \boldsymbol{\Xi}^{n}\right) \\
& =f_{h}^{n}\left(\boldsymbol{X}^{n}, \boldsymbol{\Xi}^{n}\right), \quad \forall(\boldsymbol{x}, \boldsymbol{\xi}) \in \mathcal{M}_{h} .
\end{aligned}
$$

Step 2. Time advection in velocity space. This step consists in solving the equation

$$
\begin{aligned}
\partial_{t} f+\boldsymbol{F}(t, \boldsymbol{x}, \boldsymbol{\xi}) \cdot \partial_{\boldsymbol{\xi}} f & =0, \quad t \in\left[t^{n}, t^{n+1}\right], \\
f\left(t^{n}\right) & =f_{h}^{\star} .
\end{aligned}
$$

In order to solve (26), we integrate its associated characteristic curve equation

$$
\frac{\mathrm{d} \boldsymbol{\Xi}}{\mathrm{d} t}(t)=\boldsymbol{F}(t, \boldsymbol{X}(t), \boldsymbol{\Xi}(t))
$$


over the time interval $\left[t^{n}, t^{n+1}\right]$. Then we get

$$
\begin{aligned}
\boldsymbol{\Xi}^{n+1}-\boldsymbol{\Xi}^{n}= & \int_{t^{n}}^{t^{n+1}} \boldsymbol{F}(t, \boldsymbol{X}(t), \boldsymbol{\Xi}(t)) \mathrm{d} t \\
= & \int_{t^{n}}^{t^{n+1}} \boldsymbol{F}\left(t^{n+1 / 2}, \boldsymbol{X}\left(t^{n+1 / 2}\right), \boldsymbol{\Xi}\left(t^{n+1 / 2}\right)\right) \mathrm{d} t \\
& +\mathcal{O}\left(\Delta t^{3}\right) \\
\simeq & \boldsymbol{F}\left(t^{n+1 / 2}, \boldsymbol{X}^{n+1 / 2}, \boldsymbol{\Xi}^{n+1 / 2}\right) \Delta t,
\end{aligned}
$$

where $\left(\boldsymbol{X}^{n+1 / 2}, \boldsymbol{\Xi}^{n+1}\right) \in \mathcal{M}_{h}$ and $\boldsymbol{\Xi}^{n}$ is the origin of the characteristic curve we look for. As we do not know $\boldsymbol{\Xi}^{n+1 / 2}$, we replace it by the second order approximation in time $\boldsymbol{\Xi}^{n+1 / 2}=\left\{\boldsymbol{\Xi}^{n}+\boldsymbol{\Xi}^{n+1}\right\} / 2+\mathcal{O}\left(\Delta t^{2}\right)$. Then the previous equation becomes

$$
\begin{aligned}
& \boldsymbol{\Xi}^{n+1}-\boldsymbol{\Xi}^{n} \\
& \quad=\boldsymbol{F}\left(t^{n+1 / 2}, \boldsymbol{X}^{n+1 / 2},\left\{\boldsymbol{\Xi}^{n}+\boldsymbol{\Xi}^{n+1}\right\} / 2\right) \Delta t .
\end{aligned}
$$

In the case of the nonrelativistic Vlasov model, (27) can be inverted explicitly. The solution is decomposed into a translation due to the electric field and a rotation due to the magnetic field. In contrast to the case of the relativistic Vlasov equation, we have to solve a fixed-point problem (e.g., a Newton algorithm) because of the presence of the nonlinear term $\gamma(\boldsymbol{\xi})$. Nevertheless, this fixedpoint problem can be avoided if we integrate the characteristic curves by splitting (e.g., the Strang splitting). This is due to the electric field (translation) and the magnetic field (rotation) because during the rotation it is easy to show that the term $\gamma(\boldsymbol{\xi})$ remains constant. Therefore, the new distribution function is such that

$$
\begin{aligned}
f_{h}^{\star \star}(\boldsymbol{x}, \boldsymbol{\xi}) & :=\hat{f}_{h}^{n+1 / 2}\left(\boldsymbol{X}^{n+1 / 2}, \boldsymbol{\Xi}^{n+1}\right) \\
& =f_{h}^{\star}\left(\boldsymbol{X}^{n+1 / 2}, \boldsymbol{\Xi}^{n}\right), \quad \forall(\boldsymbol{x}, \boldsymbol{\xi}) \in \mathcal{M}_{h} .
\end{aligned}
$$

Step 3. Half time advection in physical space. This step consists in solving

$$
\begin{aligned}
\partial_{t} f+\boldsymbol{v}(\boldsymbol{\xi}) \cdot \partial_{\boldsymbol{x}} f & =0, \quad t \in\left[t^{n+1 / 2}, t^{n+1}\right], \\
f\left(t^{n+1 / 2}\right) & =f_{h}^{\star \star} .
\end{aligned}
$$

In order to solve (28), we integrate its associated characteristic curve equation

$$
\frac{\mathrm{d} \boldsymbol{X}}{\mathrm{d} t}(t)=\boldsymbol{v}(\boldsymbol{\Xi}(t))
$$

over the time interval $\left[t^{n+1 / 2}, t^{n+1}\right]$. Then we get

$$
\begin{aligned}
\boldsymbol{X}^{n+1}-\boldsymbol{X}^{n+1 / 2} & =\int_{t^{n+1 / 2}}^{t^{n+1}} \boldsymbol{v}(\boldsymbol{\Xi}(t)) \mathrm{d} t \\
& =\int_{t^{n+1 / 2}}^{t^{n+1}} \boldsymbol{v}\left(\boldsymbol{\Xi}\left(t^{n+1}\right)\right) \mathrm{d} t+\mathcal{O}\left(\Delta t^{2}\right) \\
& \simeq \boldsymbol{v}\left(\boldsymbol{\Xi}^{n+1}\right) \Delta t / 2,
\end{aligned}
$$

where $\left(\boldsymbol{X}^{n+1}, \boldsymbol{\Xi}^{n+1}\right) \in \mathcal{M}_{h}$ and $\boldsymbol{X}^{n+1 / 2}$ is the origin of the characteristic curve we look for. Therefore, the new distribution function is such that

$$
\begin{aligned}
f_{h}^{n+1}(\boldsymbol{x}, \boldsymbol{\xi}) & =f_{h}^{n+1}\left(\boldsymbol{X}^{n+1}, \boldsymbol{\Xi}^{n+1}\right) \\
& =f_{h}^{\star \star}\left(\boldsymbol{X}^{n+1 / 2}, \boldsymbol{\Xi}^{n+1}\right), \quad \forall(\boldsymbol{x}, \boldsymbol{\xi}) \in \mathcal{M}_{h} .
\end{aligned}
$$

In this algorithm, which is of the second order in time (Besse, 2004; Besse and Mehrenberger, 2006), we note that there remains to compute a "good" approximation of $\boldsymbol{F}\left(t^{n+1 / 2}, \cdot, \cdot\right)$. It is the aim of the next section.

\subsection{Properties of the Vlasov-\{Darwin, Poisswell $\}$}

Models. In order to have a good approximation of the force field $\boldsymbol{F}^{n+1 / 2}=\boldsymbol{F}\left(t^{n+1 / 2}, \cdot, \cdot\right)$, we have to compute $\boldsymbol{B}^{n+1 / 2},-\nabla \phi^{n+1 / 2}, \boldsymbol{E}_{\mathrm{sol}}^{n+1 / 2}$ and $-\varepsilon \partial_{t} \boldsymbol{A}^{n+1 / 2}$.

3.2.1. Computation of $B^{n+1 / 2}$ and $-\nabla \phi^{n+1 / 2}$. Let us first compute $\partial_{t} \rho$ and $\partial_{t} \boldsymbol{j}$. If we multiply (1) by $q$ and integrate the result, we get

$$
\partial_{t} \rho=-q \int_{\mathbb{R}^{3}} \boldsymbol{v} \cdot \nabla_{\boldsymbol{x}} f \mathrm{~d} \boldsymbol{\xi} .
$$

If we multiply (1) by $q \boldsymbol{v}$ and integrate the result, then, after some algebra, we get

$\partial_{t} \boldsymbol{j}=-q \int_{\mathbb{R}^{3}} \boldsymbol{v}\left(\boldsymbol{v} \cdot \nabla_{\boldsymbol{x}} f\right) \mathrm{d} \boldsymbol{\xi}+q\left(\rho_{\gamma} \boldsymbol{E}+\varepsilon \boldsymbol{j}_{\gamma} \times \boldsymbol{B}\right)-K_{\varepsilon, 1} \boldsymbol{E}$,

where

$$
\begin{aligned}
& \rho_{\gamma^{k}}=q \int_{\mathbb{R}^{3}} f \frac{\mathrm{d} \boldsymbol{\xi}}{\gamma^{k}}, \quad j_{\gamma^{k}}=q \int_{\mathbb{R}^{3}} \boldsymbol{v} f \frac{\mathrm{d} \boldsymbol{\xi}}{\gamma^{k}}, \\
& K_{\varepsilon, k}=\varepsilon^{2} q^{2} \int_{\mathbb{R}^{3}}(\boldsymbol{v} \otimes \boldsymbol{v}) f \frac{\mathrm{d} \boldsymbol{\xi}}{\gamma^{k}}, \quad k \in \mathbb{N} .
\end{aligned}
$$

The first way to get an approximation to $\rho^{n+1 / 2}$ is to integrate with respect to $\boldsymbol{\xi}$ the distribution function $\tilde{f}^{n+1 / 2}=f^{n}(x-\boldsymbol{v} \Delta t / 2, \boldsymbol{\xi})$. Therefore, we get

$$
\rho^{n+1 / 2} \simeq q \int_{\mathbb{R}^{3}} \tilde{f}^{n+1 / 2} \mathrm{~d} \boldsymbol{\xi}
$$


Another way is to integrate (29) over the time interval $\left[t^{n}, t^{n+1 / 2}\right]$. Then we get

$$
\rho^{n+1 / 2}=\rho^{n}-q \frac{\Delta t}{2} \int_{\mathbb{R}^{3}} \boldsymbol{v} \cdot \nabla_{\boldsymbol{x}} f^{n} \mathrm{~d} \boldsymbol{\xi}+\mathcal{O}\left(\Delta t^{2}\right) .
$$

In fact, the computations of $\rho^{n+1 / 2}$ given by (32) and (33) are equivalent up to the second order in time because, if we use a Taylor expansion of $\tilde{f}^{n+1 / 2}$, then we get

$$
\tilde{f}^{n+1 / 2}=f^{n}-\frac{\Delta t}{2} \boldsymbol{v} \cdot \nabla_{\boldsymbol{x}} f^{n}+\mathcal{O}\left(\Delta t^{2}\right) .
$$

If we plug (34) into (32), then we obtain (33). Once we know $\rho^{n+1 / 2}$, we can get $\phi^{n+1 / 2}$ by solving (9) with any classical methods (spectral methods, finite difference methods, finite element methods, etc.). Next, we aim to compute $\boldsymbol{j}^{n+1 / 2}$. Let us define

$$
\tilde{\boldsymbol{j}}^{n+1 / 2}=q \int_{\mathbb{R}^{3}} \boldsymbol{v} \tilde{f}^{n+1 / 2} \mathrm{~d} \boldsymbol{\xi} .
$$

Then one way to compute $\boldsymbol{j}^{n+1 / 2}$ is

$\boldsymbol{j}^{n+1 / 2} \simeq \tilde{\boldsymbol{j}}^{n+1 / 2}+q \frac{\Delta t}{2}\left(\rho_{\gamma}^{n} \boldsymbol{E}^{n}+\varepsilon \boldsymbol{j}_{\gamma}^{n} \times \boldsymbol{B}^{n}\right)-\frac{\Delta t}{2} K_{\varepsilon, 1}^{n} \boldsymbol{E}^{n}$.

A second alternative is to integrate (30) over the time interval $\left[t^{n}, t^{n+1 / 2}\right]$. Consequently, we get

$$
\begin{aligned}
\boldsymbol{j}^{n+1 / 2}= & \boldsymbol{j}^{n}-q \frac{\Delta t}{2} \int_{\mathbb{R}^{3}} \boldsymbol{v}\left(\boldsymbol{v} \cdot \nabla_{\boldsymbol{x}} f^{n}\right) \mathrm{d} \boldsymbol{\xi} \\
& +q \frac{\Delta t}{2}\left(\rho_{\gamma}^{n} \boldsymbol{E}^{n}+\varepsilon \boldsymbol{j}_{\gamma}^{n} \times \boldsymbol{B}^{n}\right) \\
& -\frac{\Delta t}{2} K_{\varepsilon, 1}^{n} \boldsymbol{E}^{n}+\mathcal{O}\left(\Delta t^{2}\right) .
\end{aligned}
$$

Once again the approximations of $j^{n+1 / 2}$ given by (36) and (37) are equivalent up to the second order in time because, if we plug the Taylor expansion (34) of $\tilde{f}^{n+1 / 2}$ into (36), then using (35), we obtain (37). Having computed $\boldsymbol{j}^{n+1 / 2}$, we can get $\boldsymbol{B}^{n+1 / 2}$ by solving (6) with any classical numerical method. Note that in the nonrelativistic case all the terms involving $K_{\varepsilon, 1}$ disappear, $\rho_{\gamma}$ and $\boldsymbol{j}_{\gamma}$ tending to $\rho$ and $\boldsymbol{j}$, respectively.

3.2.2. Computation of $E_{\mathrm{sol}}^{n+1 / 2}$ and $-\varepsilon \partial_{t} A^{n+1 / 2}$. We only treat the case of $\boldsymbol{E}_{\mathrm{sol}}$, because from (24) we see that the case of $-\varepsilon \partial_{t} \boldsymbol{A}$ is the same as that of $\boldsymbol{E}_{\text {sol }}$ provided that we drop the term $-\varepsilon^{2} \partial_{t}^{2} \boldsymbol{E}_{\text {irr }}$ in (7). From (7) and (30) we get

$$
\begin{aligned}
-\Delta \boldsymbol{E}_{\mathrm{sol}}= & \varepsilon^{2}\left(q \int_{\mathbb{R}^{3}} \boldsymbol{v}\left(\boldsymbol{v} \cdot \nabla_{\boldsymbol{x}} f\right) \mathrm{d} \boldsymbol{\xi}-q\left(\rho_{\gamma} \boldsymbol{E}+\varepsilon \boldsymbol{j}_{\gamma} \times \boldsymbol{B}\right)\right. \\
& \left.+K_{\varepsilon, 1} \boldsymbol{E}-\partial_{t}^{2} \boldsymbol{E}_{\mathrm{irr}}\right)
\end{aligned}
$$

If we separate the solenoidal and irrotationnal parts of the electric field on the right-hand side of (38), then we obtain the following Helmholtz equation:

$$
-\Delta \boldsymbol{E}_{\mathrm{sol}}+\widetilde{K}_{\varepsilon} \boldsymbol{E}_{\mathrm{sol}}=\boldsymbol{S},
$$

where

$$
\widetilde{K}_{\varepsilon}=\varepsilon^{2}\left(q \rho_{\gamma} I-K_{\varepsilon, 1}\right)
$$

and

$$
\begin{aligned}
\boldsymbol{S}= & \varepsilon^{2} q \int_{\mathbb{R}^{3}} \boldsymbol{v}\left(\boldsymbol{v} \cdot \nabla_{\boldsymbol{x}} f\right) \mathrm{d} \boldsymbol{\xi}-\varepsilon^{2} q\left(\rho_{\gamma} \boldsymbol{E}_{\mathrm{irr}}\right. \\
& \left.+\varepsilon \boldsymbol{j}_{\gamma} \times \boldsymbol{B}\right)+\varepsilon^{2} K_{\varepsilon, 1} \boldsymbol{E}_{\mathrm{irr}}-\varepsilon^{2} \partial_{t}^{2} \boldsymbol{E}_{\mathrm{irr}} .
\end{aligned}
$$

In order to get $\boldsymbol{E}_{\mathrm{sol}}^{n+1 / 2}$, we have to solve (39) at time $t^{n+1 / 2}$ and, therefore, we have to compute $\widetilde{K}_{\varepsilon}^{n+1 / 2}$ and $\boldsymbol{S}^{n+1 / 2}$. Let us first treat the source term $\boldsymbol{S}^{n+1 / 2}$. It contains the term

$$
\begin{array}{r}
-\varepsilon^{2} q\left(\rho_{\gamma}^{n+1 / 2} \boldsymbol{E}_{\mathrm{irr}}^{n+1 / 2}+\varepsilon \boldsymbol{j}_{\gamma}^{n+1 / 2} \times \boldsymbol{B}^{n+1 / 2}\right) \\
+\varepsilon^{2} K_{\varepsilon, 1}^{n+1 / 2} \boldsymbol{E}_{\mathrm{irr}}^{n+1 / 2}
\end{array}
$$

for which we have to compute $K_{\varepsilon, 1}^{n+1 / 2}, \rho_{\gamma}^{n+1 / 2}$ and $\boldsymbol{j}_{\gamma}^{n+1 / 2}$. We shall deal with the term $K_{\varepsilon, 1}^{n+1 / 2}$ later. Let us focus on $\rho_{\gamma}^{n+1 / 2}$ and $\boldsymbol{j}_{\gamma}^{n+1 / 2}$. If we divide (1) by $\gamma$ and integrate it with respect to $\xi$ and the last term by parts, we get, as $\nabla_{\xi} \cdot \boldsymbol{F}=0$,

$$
\partial_{t}\left(\int_{\mathbb{R}^{3}} f \frac{\mathrm{d} \boldsymbol{\xi}}{\gamma}\right)=-\int_{\mathbb{R}^{3}} \boldsymbol{v} \cdot \nabla_{\boldsymbol{x}} f \frac{\mathrm{d} \boldsymbol{\xi}}{\gamma}-\varepsilon^{2} \int_{\mathbb{R}^{3}} \boldsymbol{F} \cdot \boldsymbol{v} f \frac{\mathrm{d} \boldsymbol{\xi}}{\gamma^{2}} .
$$

Integrating (42) over the time interval $\left[t^{n}, t^{n+1 / 2}\right]$ and using (34), we get

$$
\begin{aligned}
\rho_{\gamma}^{n+1 / 2}= & \int_{\mathbb{R}^{3}} \tilde{f}^{n+1 / 2} \frac{\mathrm{d} \boldsymbol{\xi}}{\gamma}-\varepsilon^{2} q^{2} \frac{\Delta t}{2} \int_{\mathbb{R}^{3}} \boldsymbol{E}^{n} \cdot \boldsymbol{v} f^{n} \frac{\mathrm{d} \boldsymbol{\xi}}{\gamma^{2}} \\
& +\mathcal{O}\left(\Delta t^{2}\right) \\
= & \tilde{\rho}_{\gamma}^{n+1 / 2}-\varepsilon^{2} q \frac{\Delta t}{2} j_{\gamma^{2}}^{n} \cdot \boldsymbol{E}^{n}+\mathcal{O}\left(\Delta t^{2}\right) .
\end{aligned}
$$

If we multiply (1) by $\boldsymbol{\xi} / \gamma$ and integrate the result, the last term by parts, with respect to $\boldsymbol{\xi}$, we get

$$
\begin{aligned}
\partial_{t}\left(\int_{\mathbb{R}^{3}} \boldsymbol{v} f \frac{\mathrm{d} \boldsymbol{\xi}}{\gamma}\right)= & -\int_{\mathbb{R}^{3}} \boldsymbol{v}\left(\boldsymbol{v} \cdot \nabla_{\boldsymbol{x}} f\right) \frac{\mathrm{d} \boldsymbol{\xi}}{\gamma} \\
& +\int_{\mathbb{R}^{3}}\left[I-2 \varepsilon^{2} \boldsymbol{v} \otimes \boldsymbol{v}\right] \boldsymbol{F} f \frac{\mathrm{d} \boldsymbol{\xi}}{\gamma^{2}} .
\end{aligned}
$$


Integrating (44) over the time interval $\left[t^{n}, t^{n+1 / 2}\right]$ and using (34), we get

$$
\begin{aligned}
\boldsymbol{j}_{\gamma}^{n+1 / 2}= & \int_{\mathbb{R}^{3}} \boldsymbol{v} \tilde{f}^{n+1 / 2} \frac{\mathrm{d} \boldsymbol{\xi}}{\gamma}+q \frac{\Delta t}{2}\left(\rho_{\gamma^{2}}^{n+1} \boldsymbol{E}^{n}+\varepsilon \boldsymbol{j}_{\gamma^{2}}^{n} \times \boldsymbol{B}^{n}\right) \\
& -\Delta t K_{\varepsilon, 2}^{n} \boldsymbol{E}^{n}+\mathcal{O}\left(\Delta t^{2}\right) \\
= & \tilde{\boldsymbol{j}}_{\gamma}^{n+1 / 2}+q \frac{\Delta t}{2}\left(\rho_{\gamma^{2}}^{n+1} \boldsymbol{E}^{n}+\varepsilon \boldsymbol{j}_{\gamma^{2}}^{n} \times \boldsymbol{B}^{n}\right) \\
& -\Delta t K_{\varepsilon, 2}^{n} \boldsymbol{E}^{n}+\mathcal{O}\left(\Delta t^{2}\right) .
\end{aligned}
$$

Note that $\rho_{\gamma}^{n+1 / 2}$ (resp. $\boldsymbol{j}_{\gamma}^{n+1 / 2}$ ) is the same as $\rho^{n+1 / 2}$ (resp. $\boldsymbol{j}^{n+1 / 2}$ ) in the nonrelativistic case. Let us now look at the term $-\varepsilon^{2} \partial_{t}^{2} \boldsymbol{E}_{\text {irr }}^{n+1 / 2}$. One way is to use a finite difference scheme of the second order in time, $\partial_{t}^{2} E_{\mathrm{irr}}^{n+1 / 2}$

$$
=\frac{2 E_{\mathrm{irr}}^{n+1 / 2}-5 E_{\mathrm{irr}}^{n}+4 E_{\mathrm{irr}}^{n-1 / 2}-E_{\mathrm{irr}}^{n-1}}{2 \Delta t^{2}}+\mathcal{O}\left(\Delta t^{2}\right) .
$$

An alternative is to take a central finite difference scheme in time in (8), as was done in (Borodachev, 2005). Then we have

$$
\boldsymbol{j}_{\mathrm{irr}}^{n+1 / 2}+\frac{\boldsymbol{E}_{\mathrm{irr}}^{n+1 / 2}-\boldsymbol{E}_{\mathrm{irr}}^{n-1 / 2}}{\Delta t}=0 .
$$

Now we look for the equivalent equation of the scheme (46) by studying its consistency. To this end, we plug the Taylor expansion

$$
\begin{aligned}
& \boldsymbol{E}_{\mathrm{irr}}^{n-1 / 2} \\
& =\boldsymbol{E}_{\mathrm{irr}}^{n+1 / 2}-\Delta t \partial_{t} \boldsymbol{E}_{\mathrm{irr}}^{n+1 / 2}+\frac{\Delta t^{2}}{2} \partial_{t}^{2} \boldsymbol{E}_{\mathrm{irr}}^{n+1 / 2}+\mathcal{O}\left(\Delta t^{3}\right)
\end{aligned}
$$

into (46), and we obtain

$$
\begin{aligned}
& \frac{2}{\Delta t}\left(j_{\mathrm{irr}}^{n+1 / 2}+\frac{\boldsymbol{E}_{\mathrm{irr}}^{n+1 / 2}-\boldsymbol{E}_{\mathrm{irr}}^{n-1 / 2}}{\Delta t}\right) \\
&=\partial_{t}^{2} \boldsymbol{E}_{\mathrm{irr}}^{n+1 / 2}+\mathcal{O}\left(\Delta t^{2}\right),
\end{aligned}
$$

which gives an approximation of $\partial_{t}^{2} \boldsymbol{E}_{\mathrm{irr}}^{n+1 / 2}$ of the second order in time. In order to apply (47), we have to compute $j_{\text {irr }}^{n+1 / 2}$. This can be done in the following way: As the current can be decomposed as $\boldsymbol{j}=\boldsymbol{j}_{\text {irr }}+\boldsymbol{j}_{\text {sol }}$ with $\nabla$. $\boldsymbol{j}_{\text {sol }}=0$ and $\nabla \times \boldsymbol{j}_{\text {irr }}=0$, we get

$$
\begin{aligned}
0 & =\nabla \times \nabla \times \boldsymbol{j}_{\text {irr }}=-\Delta \boldsymbol{j}_{\text {irr }}+\nabla\left(\nabla \cdot \boldsymbol{j}_{\text {irr }}\right) \\
& =-\Delta \boldsymbol{j}_{\text {irr }}+\nabla(\nabla \cdot \boldsymbol{j}) .
\end{aligned}
$$

Thus, to obtain $j_{\text {irr }}^{n+1 / 2}$, we have to solve the elliptic problem

$$
\Delta \boldsymbol{j}_{\mathrm{irr}}^{n+1 / 2}=\nabla\left(\nabla \cdot \boldsymbol{j}^{n+1 / 2}\right)
$$

with any classical numerical method. Now let us compute the term

$$
\varepsilon^{2} q \int_{\mathbb{R}^{3}} \boldsymbol{v}\left(\boldsymbol{v} \cdot \nabla_{\boldsymbol{x}} f^{n+1 / 2}\right) \mathrm{d} \boldsymbol{\xi} .
$$

If we take the gradient of the Vlasov equation (1) with respect to $\boldsymbol{x}$, take the scalar product with the velocity $\boldsymbol{v}$, multiply the result by $\boldsymbol{v}$ and integrate with respect to $\boldsymbol{\xi}$, then after some algebra (integration by parts) we get

$$
\begin{aligned}
\partial_{t}\left(\int_{\mathbb{R}^{3}} \boldsymbol{v}\left(\boldsymbol{v} \cdot \nabla_{\boldsymbol{x}} f\right) \mathrm{d} \boldsymbol{\xi}\right) \\
=-\int_{\mathbb{R}^{3}} \boldsymbol{v}\left(\boldsymbol{v}^{T} \nabla_{\boldsymbol{x}}^{2} f \boldsymbol{v}\right) \mathrm{d} \boldsymbol{\xi}+\int_{\mathbb{R}^{3}} \boldsymbol{v} \nabla_{\boldsymbol{x}} \cdot(\boldsymbol{F} f) \frac{\mathrm{d} \boldsymbol{\xi}}{\gamma} \\
\quad+\int_{\mathbb{R}^{3}} \nabla_{\boldsymbol{x}}^{T}(\boldsymbol{F} f) \boldsymbol{v} \frac{\mathrm{d} \boldsymbol{\xi}}{\gamma} \\
\quad-2 \varepsilon^{2} \int_{\mathbb{R}^{3}} \boldsymbol{v} \otimes \boldsymbol{v}: \nabla_{\boldsymbol{x}}(\boldsymbol{F} f) \boldsymbol{v} \frac{\mathrm{d} \boldsymbol{\xi}}{\gamma} .
\end{aligned}
$$

If we integrate (49) over the time interval $\left[t^{n}, t^{n+1 / 2}\right]$, we obtain

$$
\begin{aligned}
\int_{\mathbb{R}^{3}} \boldsymbol{v}\left(\boldsymbol{v} \cdot \nabla_{\boldsymbol{x}} f^{n+1 / 2}\right) \mathrm{d} \boldsymbol{\xi} \\
=\int_{\mathbb{R}^{3}} \boldsymbol{v}\left(\boldsymbol{v} \cdot \nabla_{\boldsymbol{x}} f^{n}\right) \mathrm{d} \xi-\frac{\Delta t}{2} \int_{\mathbb{R}^{3}} \boldsymbol{v}\left(\boldsymbol{v}^{T} \nabla_{\boldsymbol{x}}^{2} f^{n} \boldsymbol{v}\right) \mathrm{d} \boldsymbol{\xi} \\
\quad+\frac{\Delta t}{2} \int_{\mathbb{R}^{3}}\left[\boldsymbol{v} \nabla_{\boldsymbol{x}} \cdot\left(\boldsymbol{F}^{n} f^{n}\right)+\nabla_{\boldsymbol{x}}^{T}\left(\boldsymbol{F}^{n} f^{n}\right) \boldsymbol{v}\right] \frac{\mathrm{d} \boldsymbol{\xi}}{\gamma} \\
\quad-\varepsilon^{2} \Delta t \int_{\mathbb{R}^{3}} \boldsymbol{v} \otimes \boldsymbol{v}: \nabla_{\boldsymbol{x}}\left(\boldsymbol{F}^{n} f^{n}\right) \boldsymbol{v} \frac{\mathrm{d} \boldsymbol{\xi}}{\gamma}+\mathcal{O}\left(\Delta t^{2}\right),
\end{aligned}
$$

which is equivalent up to the second order in time to

$$
\begin{aligned}
\int_{\mathbb{R}^{3}} \boldsymbol{v}\left(\boldsymbol{v} \cdot \nabla_{\boldsymbol{x}} f^{n+1 / 2}\right) \mathrm{d} \boldsymbol{\xi} \\
=\int_{\mathbb{R}^{3}} \boldsymbol{v}\left(\boldsymbol{v} \cdot \nabla_{\boldsymbol{x}} \tilde{f}^{n+1 / 2}\right) \mathrm{d} \boldsymbol{\xi} \\
\quad+\frac{\Delta t}{2} \int_{\mathbb{R}^{3}} \boldsymbol{v} \nabla_{\boldsymbol{x}} \cdot\left(\boldsymbol{F}^{n} f^{n}\right) \frac{\mathrm{d} \boldsymbol{\xi}}{\gamma} \\
\quad+\frac{\Delta t}{2} \int_{\mathbb{R}^{3}} \nabla_{\boldsymbol{x}}^{T}\left(\boldsymbol{F}^{n} f^{n}\right) \boldsymbol{v} \frac{\mathrm{d} \boldsymbol{\xi}}{\gamma} \\
\quad-\varepsilon^{2} \Delta t \int_{\mathbb{R}^{3}} \boldsymbol{v} \otimes \boldsymbol{v}: \nabla_{\boldsymbol{x}}\left(\boldsymbol{F}^{n} f^{n}\right) \boldsymbol{v} \frac{\mathrm{d} \boldsymbol{\xi}}{\gamma}+\mathcal{O}\left(\Delta t^{2}\right) .
\end{aligned}
$$

Note that in (50) we have used (34), and that the last integral term in (50) disappears in the nonrelativistic case. Equation (50) involves only the gradients of the electromagnetic field which can be evaluated by finite difference or finite element methods and some moments in the velocity of the distribution function and its gradients 
with respect to $x$. Those can be evaluated by differentiating the interpolation operator used to approximate the distribution function and replacing the velocity integrals by some quadrature formulae. In (39) and (41), we have to compute $K_{\varepsilon, 1}$ at time $t^{n+1 / 2}$, where its definition is given in (31). If we multiply the Vlasov equation (1) by $\boldsymbol{v} \otimes \boldsymbol{v} / \gamma$ and integrate the result with respect to $\xi$, then after some algebra (integration by parts) we get

$$
\begin{aligned}
\partial_{t}\left(\int_{\mathbb{R}^{3}} \boldsymbol{v} \otimes \boldsymbol{v} f \frac{\mathrm{d} \boldsymbol{\xi}}{\gamma}\right) \\
=-\int_{\mathbb{R}^{3}}(\boldsymbol{v} \otimes \boldsymbol{v})\left(\boldsymbol{v} \cdot \nabla_{\boldsymbol{x}} f\right) \frac{\mathrm{d} \boldsymbol{\xi}}{\gamma} \\
\quad+\int_{\mathbb{R}^{3}}(\boldsymbol{v} \otimes \boldsymbol{F}+\boldsymbol{v} \otimes \boldsymbol{F} \\
\left.-3 \varepsilon^{2}(\boldsymbol{v} \otimes \boldsymbol{v})(\boldsymbol{F} \cdot \boldsymbol{v})\right) f \frac{\mathrm{d} \boldsymbol{\xi}}{\gamma^{2}} .
\end{aligned}
$$

Using (34), the integration of (51) over the time interval $\left[t^{n}, t^{n+1 / 2}\right]$ gives

$$
\begin{aligned}
\int_{\mathbb{R}^{3}} \boldsymbol{v} \otimes & \boldsymbol{v} f^{n+1 / 2} \frac{\mathrm{d} \boldsymbol{\xi}}{\gamma} \\
= & \int_{\mathbb{R}^{3}} \boldsymbol{v} \otimes \boldsymbol{v} \tilde{f}^{n+1 / 2} \frac{\mathrm{d} \boldsymbol{\xi}}{\gamma} \\
& +\frac{\Delta t}{2} \int_{\mathbb{R}^{3}}\left(\boldsymbol{v} \otimes \boldsymbol{F}^{n}+\boldsymbol{v} \otimes \boldsymbol{F}^{n}\right. \\
& \left.-3 \varepsilon^{2}(\boldsymbol{v} \otimes \boldsymbol{v})\left(\boldsymbol{F}^{n} \cdot \boldsymbol{v}\right)\right) f^{n} \frac{\mathrm{d} \boldsymbol{\xi}}{\gamma^{2}}+\mathcal{O}\left(\Delta t^{2}\right) .
\end{aligned}
$$

Note that the term $K_{\varepsilon, 1}^{n+1 / 2}$ disappears in the nonrelativistic case.

3.3. Complete Numerical Scheme. In this section we summarize the complete algorithm for the approximation of the Vlasov-Darwin and Vlasov-Poisswell models. Let us suppose that we know $f_{h}^{n}$ being an approximation of the distribution function $f$ at time $t^{n}$, and for the VlasovDarwin system we only suppose in addition that we know approximations of the irrotationnal part of the electric field at time $t^{n-1 / 2}, \boldsymbol{E}_{\mathrm{irr}, h}^{n-1 / 2}$ and at time $t^{n-1}, \boldsymbol{E}_{\mathrm{irr}, h}^{n-1}$.

Step 1. Compute:

$$
\rho_{\gamma^{k}, h}^{n}=q \int_{\mathbb{R}^{3}} f_{h}^{n} \frac{\mathrm{d} \boldsymbol{\xi}}{\gamma^{k}}, \quad j_{\gamma^{k}, h}^{n}=q \int_{\mathbb{R}^{3}} \boldsymbol{v} f_{h}^{n} \frac{\mathrm{d} \boldsymbol{\xi}}{\gamma^{k}}, \quad k \in\{0,1,2\} .
$$

Solve:

$$
\begin{aligned}
\boldsymbol{E}_{\mathrm{irr}, h}^{n} & =\nabla \Delta^{-1} \rho_{h}^{n}, \quad \boldsymbol{B}_{h}^{n}=-\varepsilon \Delta^{-1} \nabla \times \boldsymbol{j}_{h}^{n}, \\
\boldsymbol{j}_{\mathrm{irr}, h}^{n} & =\Delta^{-1} \nabla\left(\nabla \cdot \boldsymbol{j}_{h}^{n}\right) .
\end{aligned}
$$

Compute:

$$
\begin{aligned}
K_{\varepsilon, k}^{n}= & \varepsilon^{2} q^{2} \int_{\mathbb{R}^{3}}(\boldsymbol{v} \otimes \boldsymbol{v}) f_{h}^{n} \frac{\mathrm{d} \boldsymbol{\xi}}{\gamma^{k}}, \quad k \in\{1,2\}, \\
\widetilde{K}_{\varepsilon}^{n}= & \varepsilon^{2}\left(q \rho_{\gamma, h}^{n} I-K_{\varepsilon, 1}^{n}\right), \\
S_{D}^{n}= & \varepsilon^{2} q \int_{\mathbb{R}^{3}} \boldsymbol{v}\left(\boldsymbol{v} \cdot \nabla_{\boldsymbol{x}} f_{h}^{n}\right) \mathrm{d} \boldsymbol{\xi} \\
& -\varepsilon^{2} q\left(\rho_{\gamma, h}^{n} \boldsymbol{E}_{\mathrm{irr}, h}^{n}+\varepsilon \boldsymbol{j}_{\gamma, h}^{n} \times \boldsymbol{B}_{h}^{n}\right) \\
& +K_{\varepsilon, 1}^{n} \boldsymbol{E}_{\mathrm{irr}, h}^{n} \\
& -\varepsilon^{2} \frac{2}{\Delta t}\left(\boldsymbol{j}_{\mathrm{irr}, h}^{n}+\frac{\boldsymbol{E}_{\mathrm{irr}, h}^{n}-\boldsymbol{E}_{\mathrm{irr}, h}^{n-1}}{\Delta t}\right), \\
S_{P}^{n}= & \varepsilon^{2} q \int_{\mathbb{R}^{3}} \boldsymbol{v}\left(\boldsymbol{v} \cdot \nabla_{\boldsymbol{x}} f_{h}^{n}\right) \mathrm{d} \boldsymbol{\xi} \\
& -\varepsilon^{2} q\left(\rho_{\gamma, h}^{n} \boldsymbol{E}_{\mathrm{irr}, h}^{n}+\varepsilon \boldsymbol{j}_{\gamma, h}^{n} \times \boldsymbol{B}_{h}^{n}\right) \\
& +K_{\varepsilon, 1}^{n} \boldsymbol{E}_{\mathrm{irr}, h}^{n},
\end{aligned}
$$

Solve:

$$
\begin{aligned}
\boldsymbol{E}_{\text {sol, } h}^{n} & =\left(-\Delta+\tilde{K}_{\varepsilon}^{n}\right)^{-1} S_{D}^{n}, \\
-\varepsilon \partial_{t} \boldsymbol{A}_{h}^{n} & =\left(-\Delta+\tilde{K}_{\varepsilon}^{n}\right)^{-1} S_{P}^{n} .
\end{aligned}
$$

Option (a): here we can compute $\boldsymbol{j}_{h}^{n+1 / 2}, \boldsymbol{j}_{\gamma, h}^{n+1 / 2}, \rho_{\gamma, h}^{n+1 / 2}$ as

$$
\begin{aligned}
\boldsymbol{j}_{h}^{n+1 / 2}= & \boldsymbol{j}_{h}^{n}-q \frac{\Delta t}{2} \int_{\mathbb{R}^{3}} \boldsymbol{v}\left(\boldsymbol{v} \cdot \nabla_{\boldsymbol{x}} f_{h}^{n}\right) \mathrm{d} \boldsymbol{\xi} \\
& +q \frac{\Delta t}{2}\left(\rho_{\gamma, h}^{n} \boldsymbol{E}_{h}^{n}+\varepsilon \boldsymbol{j}_{\gamma, h}^{n} \times \boldsymbol{B}_{h}^{n}\right)-\frac{\Delta t}{2} K_{\varepsilon, 1}^{n} \boldsymbol{E}_{h}^{n}, \\
\rho_{\gamma, h}^{n+1 / 2}= & \rho_{\gamma, h}^{n}-q \frac{\Delta t}{2} \int_{\mathbb{R}^{3}} \boldsymbol{v} \cdot \nabla_{\boldsymbol{x}} f_{h}^{n} \frac{\mathrm{d} \boldsymbol{\xi}}{\gamma} \\
& -\varepsilon^{2} q \frac{\Delta t}{2} \boldsymbol{j}_{\gamma^{2}, h}^{n} \cdot \boldsymbol{E}_{h}^{n}, \\
\boldsymbol{j}_{\gamma, h}^{n+1 / 2}= & \boldsymbol{j}_{\gamma, h}^{n}-q \frac{\Delta t}{2} \int_{\mathbb{R}^{3}} \boldsymbol{v}\left(\boldsymbol{v} \cdot \nabla_{\boldsymbol{x}} f_{h}^{n}\right) \frac{\mathrm{d} \boldsymbol{\xi}}{\gamma} \\
& +q \frac{\Delta t}{2}\left(\rho_{\gamma^{2}, h}^{n} \boldsymbol{E}_{h}^{n}+\varepsilon \boldsymbol{j}_{\gamma^{2}, h}^{n} \times \boldsymbol{B}_{h}^{n}\right)-\Delta t K_{\varepsilon, 2}^{n} \boldsymbol{E}_{h}^{n} .
\end{aligned}
$$

Step 2. Perform a half-time step advection in physical space:

$\tilde{f}_{h}^{n+1 / 2}(\boldsymbol{x}, \boldsymbol{\xi})=f_{h}^{n}(\boldsymbol{x}-\boldsymbol{v}(\boldsymbol{\xi}) \Delta t / 2, \boldsymbol{\xi}), \quad \forall(\boldsymbol{x}, \boldsymbol{\xi}) \in \mathcal{M}_{h}$.

Step 3. Compute:

$\tilde{\rho}_{h}^{n+1 / 2}=q \int_{\mathbb{R}^{3}} \tilde{f}_{h}^{n+1 / 2} \mathrm{~d} \boldsymbol{\xi}, \quad \tilde{\rho}_{\gamma, h}^{n+1 / 2}=q \int_{\mathbb{R}^{3}} \tilde{f}_{h}^{n+1 / 2} \frac{\mathrm{d} \boldsymbol{\xi}}{\gamma}$, 


$$
\tilde{\boldsymbol{j}}_{\gamma^{k}, h}^{n+1 / 2}=q \int_{\mathbb{R}^{3}} \boldsymbol{v} \tilde{f}_{h}^{n+1 / 2} \frac{\mathrm{d} \boldsymbol{\xi}}{\gamma^{k}}, \quad k \in\{1,2\} .
$$

Option (b): here we can also compute $\boldsymbol{j}_{h}^{n+1 / 2}, \boldsymbol{j}_{\gamma, h}^{n+1 / 2}$, $\rho_{\gamma, h}^{n+1 / 2}$ as

$$
\begin{aligned}
\boldsymbol{j}_{h}^{n+1 / 2}= & \tilde{\boldsymbol{j}}_{\gamma, h}^{n+1 / 2}+q \frac{\Delta t}{2}\left(\rho_{\gamma, h}^{n} \boldsymbol{E}_{h}^{n}+\varepsilon \boldsymbol{j}_{\gamma, h}^{n} \times \boldsymbol{B}_{h}^{n}\right) \\
& -\frac{\Delta t}{2} K_{\varepsilon, 1}^{n} \boldsymbol{E}_{h}^{n}, \\
\rho_{\gamma, h}^{n+1 / 2}= & \tilde{\rho}_{\gamma, h}^{n+1 / 2}-\varepsilon^{2} q \frac{\Delta t}{2} \boldsymbol{j}_{\gamma^{2}, h}^{n} \cdot \boldsymbol{E}_{h}^{n}, \\
\boldsymbol{j}_{\gamma, h}^{n+1 / 2}= & \tilde{\boldsymbol{j}}_{\gamma^{2}, h}^{n+1 / 2}+q \frac{\Delta t}{2}\left(\rho_{\gamma^{2}, h}^{n} \boldsymbol{E}_{h}^{n}+\varepsilon \boldsymbol{j}_{\gamma^{2}, h}^{n} \times \boldsymbol{B}_{h}^{n}\right) \\
& -\Delta t K_{\varepsilon, 2}^{n} \boldsymbol{E}_{h}^{n} .
\end{aligned}
$$

Solve:

$$
\begin{gathered}
\boldsymbol{E}_{\mathrm{irr}, h}^{n+1 / 2}=\nabla \Delta^{-1} \rho_{h}^{n+1 / 2}, \quad \boldsymbol{B}_{h}^{n+1 / 2}=-\varepsilon \Delta^{-1} \nabla \times \boldsymbol{j}_{h}^{n+1 / 2}, \\
\boldsymbol{j}_{\mathrm{irr}, h}^{n+1 / 2}=\Delta^{-1} \nabla\left(\nabla \cdot \boldsymbol{j}_{h}^{n+1 / 2}\right) .
\end{gathered}
$$

\section{Compute:}

$K_{\varepsilon, 1}^{n+1 / 2}$ : evaluate the integral (52) by replacing $\tilde{f}^{n+1 / 2}$ by $\tilde{f}_{h}^{n+1 / 2}, f^{n}$ by $f_{h}^{n}$ and $\boldsymbol{F}^{n}$ by $\boldsymbol{F}_{h}^{n}$.

$$
\widetilde{K}_{\varepsilon}^{n+1 / 2}=\varepsilon^{2}\left(q \rho_{\gamma, h}^{n+1 / 2} I-K_{\varepsilon, 1}^{n+1 / 2}\right) .
$$

$\int_{\mathbb{R}^{3}} \boldsymbol{v}\left(\boldsymbol{v} \cdot \nabla_{\boldsymbol{x}} f_{h}^{n+1 / 2}\right) \mathrm{d} \boldsymbol{\xi}:$ evaluate the integral (50) by replacing $\tilde{f}^{n+1 / 2}$ by $\tilde{f}_{h}^{n+1 / 2}, f^{n}$ by $f_{h}^{n}$ and $\boldsymbol{F}^{n}$ by $\boldsymbol{F}_{h}^{n}$.

$$
\begin{aligned}
S_{D}^{n+1 / 2}= & \varepsilon^{2} q \int_{\mathbb{R}^{3}} \boldsymbol{v}\left(\boldsymbol{v} \cdot \nabla_{\boldsymbol{x}} f_{h}^{n+1 / 2}\right) \mathrm{d} \boldsymbol{\xi} \\
& -\varepsilon^{2} q\left(\rho_{\gamma, h}^{n+1 / 2} \boldsymbol{E}_{\mathrm{irr}, h}^{n+1 / 2}+\varepsilon \boldsymbol{j}_{\gamma, h}^{n+1 / 2} \times \boldsymbol{B}_{h}^{n+1 / 2}\right) \\
& +K_{\varepsilon, 1}^{n+1 / 2} \boldsymbol{E}_{\mathrm{irr}, h}^{n+1 / 2} \\
& -\varepsilon^{2} \frac{2}{\Delta t}\left(\boldsymbol{j}_{\mathrm{irr}, h}^{n+1 / 2}+\frac{\boldsymbol{E}_{\mathrm{irr}, h}^{n+1 / 2}-\boldsymbol{E}_{\mathrm{irr}, h}^{n-1 / 2}}{\Delta t}\right), \\
S_{P}^{n+1 / 2}= & \varepsilon^{2} q \int_{\mathbb{R}^{3}} \boldsymbol{v}\left(\boldsymbol{v} \cdot \nabla_{\boldsymbol{x}} f_{h}^{n+1 / 2}\right) \mathrm{d} \boldsymbol{\xi} \\
& -\varepsilon^{2} q\left(\rho_{\gamma, h}^{n+1 / 2} \boldsymbol{E}_{\mathrm{irr}, h}^{n+1 / 2}+\varepsilon \boldsymbol{j}_{\gamma, h}^{n+1 / 2} \times \boldsymbol{B}_{h}^{n+1 / 2}\right) \\
& +K_{\varepsilon, 1}^{n+1 / 2} \boldsymbol{E}_{\mathrm{irr}, h}^{n+1 / 2} .
\end{aligned}
$$

Solve:

$$
\begin{aligned}
\boldsymbol{E}_{\text {sol,h }}^{n+1 / 2} & =\left(-\Delta+\tilde{K}_{\varepsilon}^{n+1 / 2}\right)^{-1} S_{D}^{n+1 / 2}, \\
-\varepsilon \partial_{t} \boldsymbol{A}_{h}^{n+1 / 2} & =\left(-\Delta+\tilde{K}_{\varepsilon}^{n+1 / 2}\right)^{-1} S_{P}^{n+1 / 2} .
\end{aligned}
$$

Step 4. Perform a full-time step advection in velocity space:

Solve: search for $\xi^{\star}$ such as

$$
\boldsymbol{\xi}-\boldsymbol{\xi}^{\star}=\boldsymbol{F}_{h}^{n+1 / 2}\left(\boldsymbol{x},\left(\boldsymbol{\xi}+\boldsymbol{\xi}^{\star}\right) / 2\right) \Delta t .
$$

Compute:

$$
\hat{f}_{h}^{n+1 / 2}(\boldsymbol{x}, \boldsymbol{\xi})=\tilde{f}_{h}^{n+1 / 2}\left(\boldsymbol{x}, \boldsymbol{\xi}^{\star}\right), \quad \forall(\boldsymbol{x}, \boldsymbol{\xi}) \in \mathcal{M}_{h} .
$$

Step 5. Perform half-time step advection in physical space:

$$
\begin{aligned}
& f_{h}^{n+1}(\boldsymbol{x}, \boldsymbol{\xi}) \\
& \quad=\hat{f}_{h}^{n+1 / 2}(\boldsymbol{x}-\boldsymbol{v}(\boldsymbol{\xi}) \Delta t / 2, \boldsymbol{\xi}), \quad \forall(\boldsymbol{x}, \boldsymbol{\xi}) \in \mathcal{M}_{h} .
\end{aligned}
$$

\section{Numerical Results}

In this section we present numerical results obtained by the algorithm described in the previous section for two physical test cases. One of them is the Landau damping of longitudinal plasma waves. This test case is considered in order to demonstrate that our scheme reproduces the theoretical damping of Langmuir waves which should not be modified by taking into accout a self-consistent lowfrequency magnetic field. The second case is the electromagnetic beam-plasma instability (EMBP). Here we choose a model with a magnetic field parallel to the $z$-axis, i.e., only one nonzero component $\boldsymbol{B}=\left(B_{z}\right)$, while the electric field $\boldsymbol{E}=\left(E_{x}, E_{y}\right)$ is transversal to $B_{z}$, and particles can move in the $x-y$ plane. The electromagnetic field $\left(E_{x}, E_{y}, B_{z}\right)$ depends on the $x$ and $y$ variables while the distribution function $f=f\left(t, x, y, v_{x}, v_{y}\right)$ reflects a fourdimensional phase space. Here we choose to work in the nonrelativistic setting because $\varepsilon=L / \tau c=\bar{v} / c$ (where $\bar{v}$ is the characteristic velocity of the problem) should be sufficiently small for the Vlasov-\{Darwin, Poisswell $\}$ models to be valid. Besides, we use the B-splines interpolation to reconstruct the distribution function on the phase space grid and interpolate its values at the origin of the characteristic set.

4.1. Landau Damping Test Case. The initial condition is

$$
f(0, x, v)=\frac{1}{2 \pi}\left(1+\alpha \cos \left(k_{x} x\right) \cos \left(k_{y} y\right)\right) e^{-\frac{v_{x}^{2}+v_{y}^{2}}{2}}
$$

for all couples $(x, y)$ in the box $[0, L]^{2}$ and for all couples $\left(v_{x}, v_{y}\right)$ in the plane $\mathbb{R}^{2}$, where $\alpha=5 \cdot 10^{-2}$ is the intensity of the perturbation. The boundary conditions are periodic. The initial distribution function represents a plasma at the thermodynamical equilibrium affected by a small perturbation in physical space which will generate a small longitudinal electrostatic field. The spatial box period is 
$L=4 \pi$ and $v_{\max }=8$ is the velocity above for which we suppose that the distribution function is null. The time step $\Delta t=1 / 8$ and the final time is $T=20 \omega_{p}^{-1}$. The parameter $\varepsilon$ is equal to 0.01 . The discretization in the physical space is 64 points per direction and in the velocity space it is 128 points per direction. We observe that the electrical energy decreases exponentially in time with the rate $\gamma=-0.400$ and the pulsation $\omega=1.69$, as it is predicted by the linear Landau theory. We observe that the Vlasov-Darwin and Vlasov-Poisswell models produce the same results. The Landau damping is a kinetic effect which takes place where the phase velocity of the electrostatic field is close to the thermal velocity of the particles. It consists in an exchange of energy from the electrostatic waves to the particles (at least in the linear regime) when the phase velocity and the thermal velocity are of the same orders. Therefore, the Vlasov-Darwin and Vlasov-Poisswell models should reproduce the same behavior (Fig. 1).

\subsection{Electromagnetic Beam-Plasma Instability Test} Case. The initial condition is

$$
\begin{aligned}
f(0, x, v) & \\
= & \frac{1}{2 \pi}\left(1+\alpha \cos \left(k_{x} x\right) \cos \left(k_{y} y\right)\right) \\
& \times\left(\frac{n_{0,1}}{v_{t h, 1}{ }^{2}} e^{-\frac{\left(v_{x}-v_{0,1}\right)^{2}+v_{y}^{2}}{2 v_{t h, 1}^{2}}}+\frac{n_{0,2}}{v_{t h, 2}{ }^{2}} e^{-\frac{\left(v_{x}-v_{0,2}\right)^{2}+v_{y}^{2}}{2 v_{t h, 2}{ }^{2}}}\right)
\end{aligned}
$$

for all pairs $(x, y)$ in the box $[0, L]^{2}$ and for all pairs $\left(v_{x}, v_{y}\right)$ in the plane $\mathbb{R}^{2}$, where $v_{0,1}$ and $v_{0,2}$ are the drift velocity of the beam and the drift velocity of the plasma, respectively. Here $v_{t h, 1}$ and $v_{t h, 2}$ are the thermal velocities, and $n_{0,1}$ and $n_{0,2}$ are the initial densities of the beam and the plasma, respectively. The boundary conditions are periodic. The paratieters $n_{0,1}, n_{0,2}, v_{0,1}$ and $v_{0,2}$ are chosen such that the total density is one and the total net current is zero. Then we have

$$
n_{0,1}+n_{0,2}=1, \quad n_{0,1} v_{0,1}+n_{0,2} v_{0,2}=0 .
$$

We suppose that the initial densities are homogeneous, i.e., $n_{0,1}$ and $n_{0,2}$ do not depend on physical space variables. This initial condition models two initially interpenetrating currents, one carried by a fast electrond beam and the other carried by a cold plasma. In order to get the theoritical growth rate of this instability, we start with the fluid equation for the motion of the particles:

$\partial_{t} \rho_{a}+\nabla \cdot \boldsymbol{j}_{a}=0, \quad \partial_{t} \boldsymbol{\xi}_{a}+\left(\boldsymbol{\xi}_{a} \cdot \nabla\right) \boldsymbol{\xi}_{a}=q\left(\boldsymbol{E}+\varepsilon \boldsymbol{v}_{a} \times \boldsymbol{B}\right)$,

where $\boldsymbol{j}_{a}=q n_{a} \boldsymbol{v}_{a}=\rho_{a} \boldsymbol{v}_{a}$, with $a=\{1,2\}$. The fluid equations are coupled to the electromagnetic field by using the Darwin or the Poisswell model. After linearizing the model formed by the nonrelativistic fluid equation and the electromagnetic field equations, using $q=-1$,

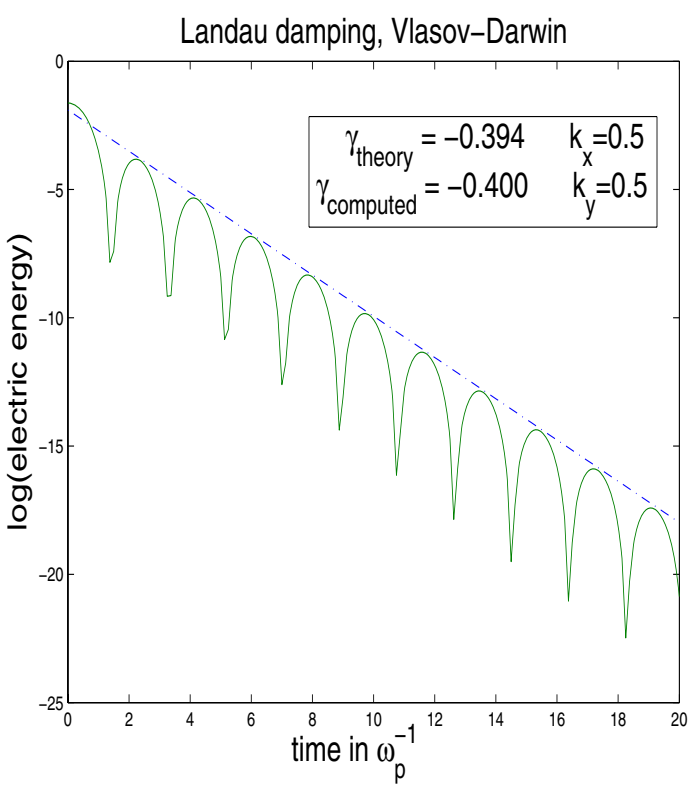

(a)

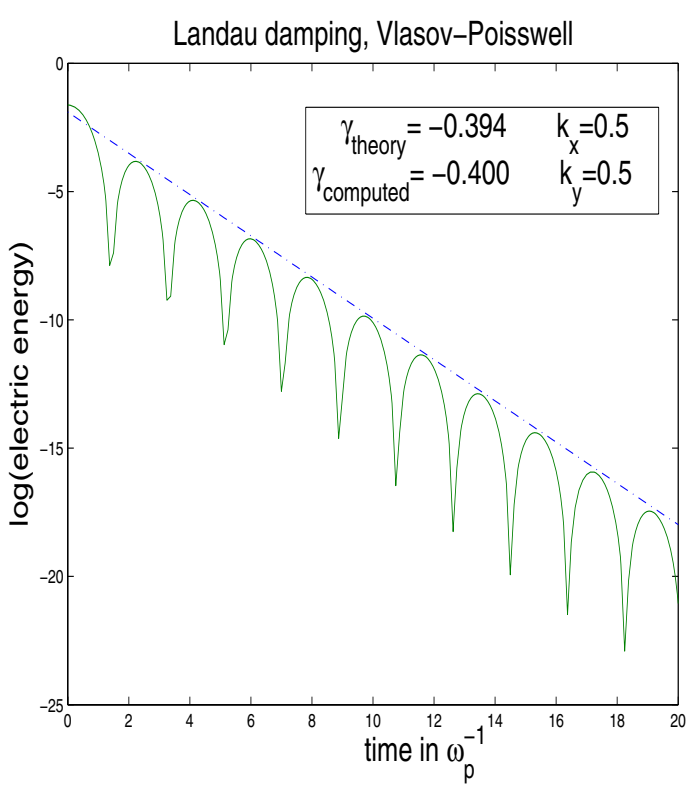

(b)

Fig. 1. Evolution of the logarithm of the electric energy in the case of the Landau damping: (a) VlasovDarwin, (b)Vlasov-Poisswell.

$\boldsymbol{j}=\sum_{a} \boldsymbol{j}_{a}$ and $\rho=\sum_{a} \rho_{a}$, the growth rate of the EMBP instability for the Vlasov-Darwin system is determined by the imaginary parts of the zeros (in the variable $\omega$ ) of the polynomial defined as the determinant of the dispersion matrix (??). In the case of the Vlasov-Poisswell model the dispersion matrix is given by (53).

We take $L=4 \pi\left(k_{x}=0.5, k_{y}=0.5\right), \varepsilon=0.01$ and $\Delta t=1 / 4$. For the symmetric case we take the parameters $n_{0,1}=n_{0,2}=0.5, v_{0,1}=-v_{0,2}=1$, $v_{t h, 1}=v_{t h, 2}=0.1, \alpha=10^{-10}$, and $v_{\max }=3$. The 


$$
\mathbb{D}_{d}\left(B_{0}, \rho_{0, a}, \boldsymbol{v}_{0, a}, \omega, \boldsymbol{k}\right)=\left(\begin{array}{cccc}
i\left(\boldsymbol{k} \cdot \boldsymbol{v}_{0,1}-\omega\right) & 0 & \varepsilon B_{0} & 0 \\
0 & i\left(\boldsymbol{k} \cdot \boldsymbol{v}_{0,2}-\omega\right) & 0 & \varepsilon B_{0} \\
-\varepsilon B_{0} & 0 & i\left(\boldsymbol{k} \cdot \boldsymbol{v}_{0,1}-\omega\right) & 0 \\
0 & -\varepsilon B_{0} & 0 & i\left(\boldsymbol{k} \cdot \boldsymbol{v}_{0,2}-\omega\right) \\
-\rho_{0,1} k_{x} & 0 & -\rho_{0,1} k_{y} & 0 \\
0 & -\rho_{0,2} k_{x} & 0 & -\rho_{0,2} k_{y} \\
i \varepsilon^{2} \rho_{0,1} \omega & i \varepsilon^{2} \rho_{0,2} \omega & 0 & 0 \\
0 & 0 & i \varepsilon^{2} \rho_{0,1} \omega & i \varepsilon^{2} \rho_{0,2} \omega \\
0 & 0 & 0 & 0 \\
0 & 0 & 0 & 0 \\
-i \varepsilon k_{y} \rho_{0,1} & -i \varepsilon k_{y} \rho_{0,2} & i \varepsilon k_{x} \rho_{0,1} & i \varepsilon k_{x} \rho_{0,2}
\end{array}\right.
$$

$\left.\begin{array}{ccccccc}0 & 0 & 1 & 0 & 1 & 0 & \varepsilon \boldsymbol{v}_{0,1_{y}} \\ 0 & 0 & 1 & 0 & 1 & 0 & \varepsilon \boldsymbol{v}_{0,2_{y}} \\ 0 & 0 & 0 & 1 & 0 & 1 & -\varepsilon \boldsymbol{v}_{0,1_{x}} \\ 0 & 0 & 0 & 1 & 0 & 1 & -\varepsilon \boldsymbol{v}_{0,2_{x}} \\ \boldsymbol{k} \cdot \boldsymbol{v}_{0,1}-\omega & 0 & 0 & 0 & 0 & 0 & 0 \\ 0 & \boldsymbol{k} \cdot \boldsymbol{v}_{0,2}-\omega & 0 & 0 & 0 & 0 & 0 \\ i \varepsilon^{2} v_{0,1_{x}} \omega & i \varepsilon^{2} v_{0,2_{x}} \omega & -|\boldsymbol{k}|^{2} & 0 & \varepsilon^{2} \omega^{2} & 0 & 0 \\ i \varepsilon^{2} v_{0,1_{y}} \omega & i \varepsilon^{2} v_{0,2_{y}} \omega & 0 & -|\boldsymbol{k}|^{2} & 0 & \varepsilon^{2} \omega^{2} & 0 \\ 1 & 1 & 0 & 0 & -i k_{x} & -i k_{y} & 0 \\ 0 & 0 & 0 & 0 & -k_{y} & k_{x} & 0 \\ i \varepsilon \boldsymbol{k} \times v_{0,1} & i \varepsilon \boldsymbol{k} \times v_{0,2} & 0 & 0 & 0 & 0 & -|\boldsymbol{k}|^{2}\end{array}\right)$.

$\mathbb{D}_{p}\left(\rho_{0, a}, \boldsymbol{v}_{0, a}, \omega, \boldsymbol{k}\right)=\left(\begin{array}{cccc}\boldsymbol{k} \cdot \boldsymbol{v}_{0,1}-\omega & 0 & 0 & 0 \\ 0 & \boldsymbol{k} \cdot \boldsymbol{v}_{0,1}-\omega & 0 & 0 \\ 0 & 0 & \boldsymbol{k} \cdot \boldsymbol{v}_{0,1}-\omega & 0 \\ 0 & 0 & 0 & \boldsymbol{k} \cdot \boldsymbol{v}_{0,1}-\omega \\ \rho_{0,1} k_{x} & 0 & \rho_{0,1} k_{y} & 0 \\ 0 & \rho_{0,2} k_{x} & 0 & \rho_{0,2} k_{y} \\ 0 & 0 & 0 & 0 \\ \varepsilon \rho_{0,1} & \varepsilon \rho_{0,2} & 0 & 0 \\ 0 & 0 & \varepsilon \rho_{0,1} & \varepsilon \rho_{0,2}\end{array}\right.$

$$
\left.\begin{array}{ccccc}
0 & 0 & -k_{x} & -\varepsilon k_{y} v_{0,1_{y}} & \varepsilon k_{x} v_{0,1_{y}} \\
0 & 0 & -k_{x} & -\varepsilon k_{y} v_{0,2_{y}} & \varepsilon k_{x} v_{0,2_{y}} \\
0 & 0 & -k_{y} & \varepsilon k_{y} v_{0,1_{x}} & -\varepsilon k_{x} v_{0,1_{x}} \\
0 & 0 & -k_{y} & \varepsilon k_{y} v_{0,2_{x}} & -\varepsilon k_{x} v_{0,2_{x}} \\
\boldsymbol{k} \cdot \boldsymbol{v}_{0,1}-\omega & 0 & 0 & 0 & 0 \\
0 & \boldsymbol{k} \cdot \boldsymbol{v}_{0,2}-\omega & 0 & 0 & 0 \\
1 & 1 & -|\boldsymbol{k}|^{2} & 0 & 0 \\
\varepsilon v_{0,1_{x}} & \varepsilon v_{0,2_{x}} & 0 & -|\boldsymbol{k}|^{2} & 0 \\
\varepsilon v_{0,1_{y}} & \varepsilon v_{0,2_{y}} & 0 & 0 & -|\boldsymbol{k}|^{2}
\end{array}\right)
$$


EMBP instability, symmetric case, Vlasov-Darwin

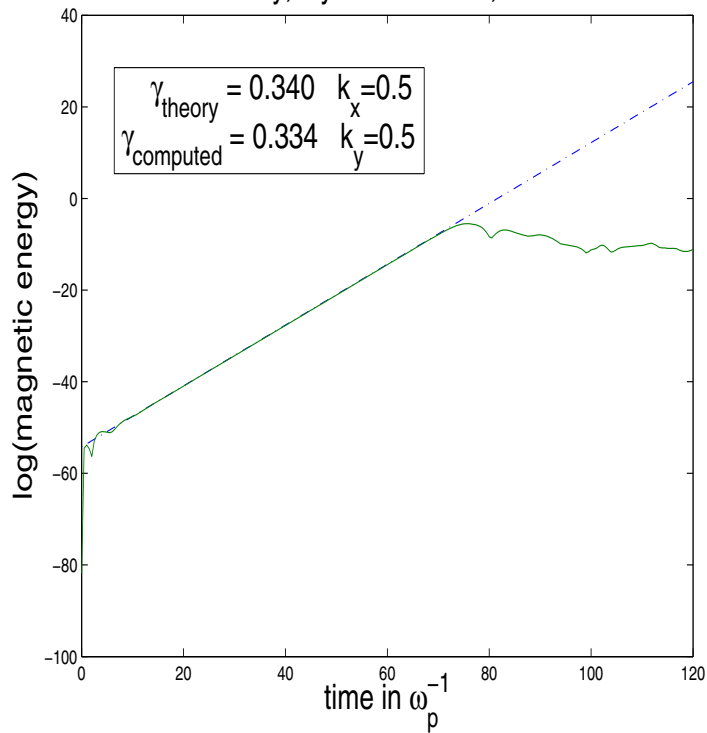

(a)

EMBP instability, symmetric case, Vlasov-Poisswell

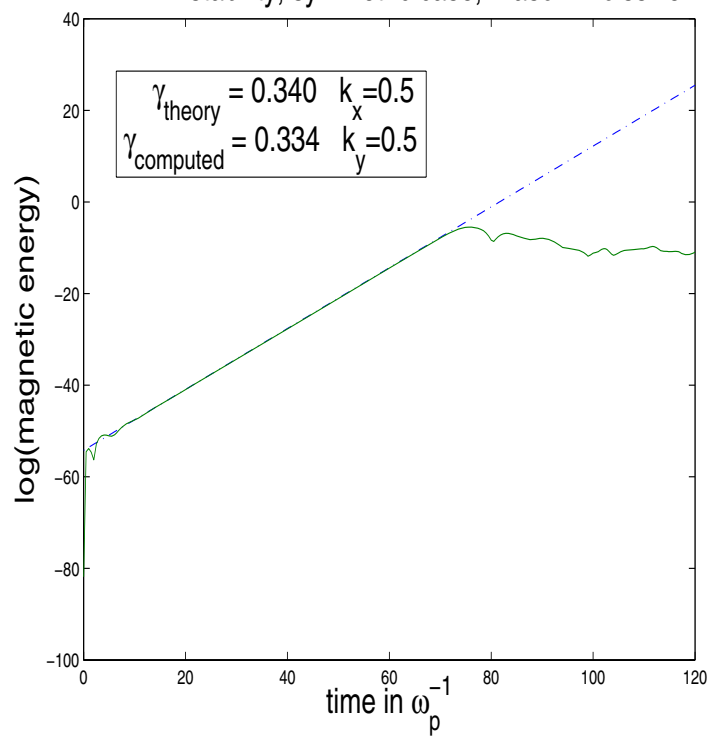

(b)

Fig. 2. Evolution of the logarithm of the magnetic energy in the case of symmetric electromagnetic beam-plasma instability: (a) Vlasov-Darwin, (b) Vlasov-Poisswell.

discretization in the physical space is 64 points per direction and in the velocity space it is 128 points per direction. In order to get the fluid growth rate of the electromagnetic beam plasma instability we solve the equation (in the variable $\omega) \operatorname{det}\left(\mathbb{D}_{d}\left(B_{0}, \rho_{0, a}, \boldsymbol{v}_{0, a}, \omega, \boldsymbol{k}\right)\right)=0$ for the Vlasov-Darwin model and $\operatorname{det}\left(\mathbb{D}_{p}\left(\rho_{0, a}, \boldsymbol{v}_{0, a}, \omega, \boldsymbol{k}\right)\right)=0$ for the Vlasov-Poisswell system. Then we find that the nonzero imaginary parts of the roots are for both systems $\gamma=0.340$. For the nonsymmetric case we take the parameters $n_{0,1}=10 / 11, n_{0,2}=1 / 11, v_{0,1}=-0.1$ $v_{0,2}=1, v_{t h, 1}=v_{t h, 2}=0.05, \alpha=10^{-6}$ and $v_{\max }=2$.
The discretization in the physical space is 64 points per direction and in the velocity space it is 256 points per direction. The growth rate of the electromagnetic beam-plasma instabilty is for both system $\gamma=0.161$.

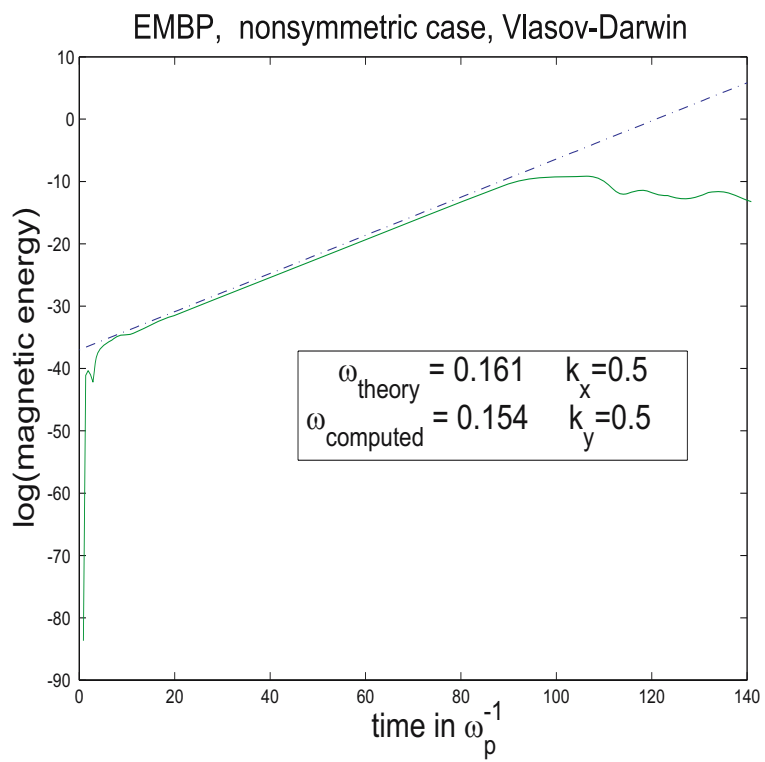

(a)

EMBP, nonsymmetric case, Vlasov-Poisswell

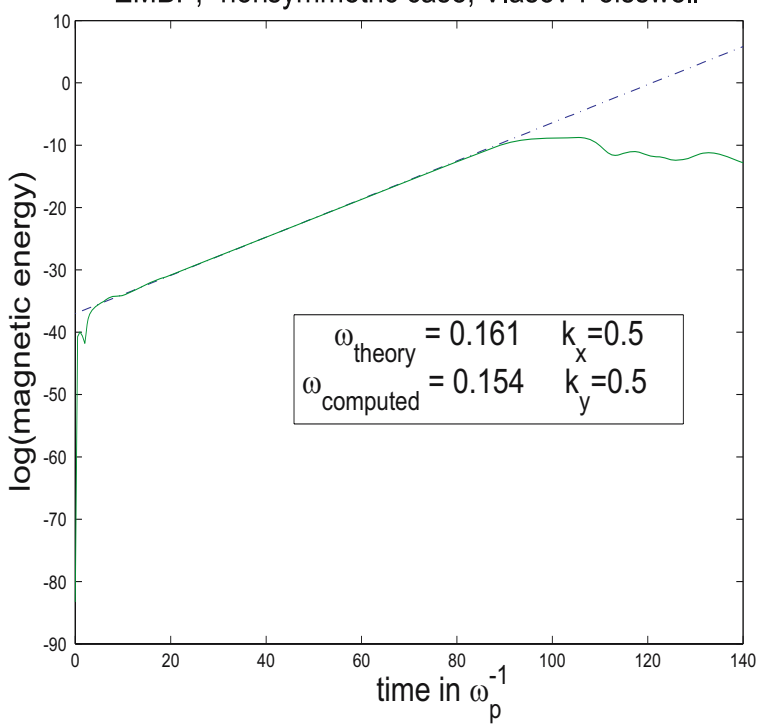

(b)

Fig. 3. Evolution of the logarithm of the magnetic energy in the case of nonsymmetric electromagnetic beam-plasma instability: (a) Vlasov-Darwin, (b) Vlasov-Poisswell.

\section{Conclusion}

We have introduced and tested numerical schemes to solve the Vlasov-Darwin and Vlasov-Poisswell systems as approximations of Vlasov-Maxwell systems for lowfrequency simulation of kinetic plasma. The results of the numerical simulations show that our new algorithm 
works well and yields good agreement with linear theory in a four-dimensional phase space code. In the future we intend to perform two-dimensional simulation where vector quantities will retain all the three components. This will allow us to analyze the evolution of an initially solid cylindrical, hot electron beam propagating in an overdense plasma and study the filamention of the beam (the beam breaks up into several beamlets via a mechanism similar to a tearing instability) which appears in laser-plasma interaction problems (Taguchi et al., 2001). Moreover, this will allow us to study magnetic reconnection which is of central interest for phenomena regarding both astrophysical environments (solar flares (Kulsrud, 1998), solar windmagnetosphere interaction (Coppi et al., 1996)) and magnetically confined plasmas for fusion research (Ottaviani and Porcelli, 1993).

\section{Acknowledgments}

The authors acknowledge the financial support from the HYKE project on Hyperbolic and Kinetic Equations: Asymptotics, Numerics, Applications, funded by the European Union (project no. No HPRN-CT-2002-00282) and by the Austrian START project (FWF project no. Y-137TEC)

\section{References}

Bauer S. and Kunze M. (2005): The Darwin approximation of the relativistic Vlasov-Maxwell system. Annales Henri Poincaré, Vol. 6, No. 2, pp. 283-308.

Bégué M.L., Ghizzo A. and Bertrand P. (1999): Twodimensional Vlasov simulation of Raman scattering and plasma beatwave acceleration on parallel computers. Journal of Computational Physics, Vol. 151, No. 2, pp. 458478.

Benachour S., Filbet F., Laurençcot P. and Sonnendrücker E.(2003): Global existence for the Vlasov-Darwin system in $\mathbb{R}^{3}$ for small initial data. Mathematical Methods in the Applied Sciences., Vol. 26, No. 4, pp. 297-319.

Besse N. (2004): Convergence of a semi-Lagrangian scheme for the one-dimensional Vlasov-Poisson system. SIAM Journal on Numerical Analysis, Vol. 42, No. 1, pp. 350-382.

Besse N. and Mehrenberger M. (2006): Convergence of classes of high-order semi-Lagrangian schemes for the VlasovPoisson system. Mathematics of Computation, (in print).

Besse N. and Sonnendrücker E. (2003): Semi-Lagrangian schemes for the Vlasov equation on an unstructured mesh of phase space. Journal of Computational Physics, Vol. 191, No. 2, pp. 341-376.

Borodachev L.V. (2005): Elliptic formulation of discrete VlasovDarwin model with the implicit finite-difference representation of particle dynamics. Proceedings of 7-th International School/Symposium for Space Simulations, Kyoto, Japan.
Califano F., Prandi R., Pegoraro F. and Bulanov S.V. (1998): Nonlinear filamentation instability driven by an inhomogeneous current in a collisionless plasma. Physical Review E, Vol. 58, No. 6, pp. 19-24.

Cheng C.Z. and Knorr G. (1976): The integration of the Vlasov equation in configuration space. Journal of Computational Physics, Vol. 22, No. 3, pp. 330-351.

Coppi B., Laval G. and Pellat R. (1996): Dynamics of the geomagnetic tail. Physical Review Letters, Vol. 16, No. 26, pp. 1207-1210.

Degond P. and Raviart P.-A. (1992): An analysis of Darwin model of approximation to Maxwell's eqautions. Forum Mathematics, Vol. 4, pp. 13-44.

Fijalkow E. (1999): A numerical solution to the Vlasov equation. Computer Physics Communications, Vol. 116, No. 2, pp. 319-328.

Filbet F., Sonnendrücker E. and Bertrand P. (2000): Conservative numerical schemes for the Vlasov equation. Journal of Computational Physics, Vol. 172, No. 1, pp. 166-187.

Gibbons M.R. and Hewett D.W. (1995): The Darwin direct implicit particle-on-cell (DADIPIC) method for simulation of low frequency plasma phenomena. Journal of Computational Physics, Vol. 120, No. 2, pp. 231-247.

Gibbons M.R. and Hewett D.W. (1997): Characterization of the Darwin direct implicit particle-in-cell method an resulting guidelines for operation. Journal of Computational Physics, Vol. 130, No. 1, pp. 54-66.

Kulsrud R.M. (1998): Magnetic reconnection in a magnetohydrodynamic plasma. Physics of Plasmas, Vol. 5, No. 5, pp. 1599-1606.

Lee W.W.L., Startsev E., Hong Q. and Davidson R.C. (2001): Electromagnetic (Darwin) model for threedimensional perturbative particle simulation of high intensity beams. Proceedings of the Particle Accelerator Conference, PACS'2001, Chicago, USA, IEEE Part, Vol. 3, p. 1906.

Masmoudi N. and Mauser N.J. (2001): The selfconsistent Pauli equation. Monatshefte für Mathematik, Vol. 132, No. 1, pp. 19-24.

Omelchenko Y.A. and Sudan R.N. (1997): A 3-D Darwin-EM Hybrid PIC code for ion ring studies. Journal of Computational Physics, Vol. 133, No. 1, pp. 146-159.

Ottaviani M. and Porcelli F. (1993): Nonlinear collisionless magnetic reconnection. Physical Review Letters, Vol. 71, No. 23, pp. 3802-3805.

Pallard C. (2006): The initial value problem for the relativistic Vlasov-Darwin system. International Mathematics Research Notices, Vol. 2006, Article ID 57191, available at: DOI:100.1155/IMRN/2006/57191.

Raviart P.-A. and Sonnendrücker E. (1996): A Hierarchy of Approximate Models for the Maxwell Equations. Numerische Mathematik, Vol. 73, No. 3, pp. 329-372.

Schmitz H. and Grauer R. (2006): Darwin-Vlasov simulations of magnetised plasmas. Journal of Computational Physics, Vol. 214, No. 2, pp. 738-756. 
Sabatier M., Such N., Mineau P., Feix M., Shoucri M., Bertrand P. and Fijalkow E. (1990): Numerical simulations of the Vlasov equation using a flux conservation scheme; comparaison with the cubic spline interpolation code. Technical Report No. 330e, Centre Canadien de Fusion Magnetique, Varennes, Canada.

Sonnendrücker E., Ambrosiano J.J. and Brandon S.T. (1995): A finite element formulation of the Darwin PIC model for use on unstructured grids. Journal of Computational Physics, Vol. 121, No. 2, pp. 281-297.
Taguchi T., Antonsen T.M. Jr., Liu C.S. and Mima K. (2001): Structure formation and tearing of an MeV cylindrical electron beam in a laser-produced plasma. Physical Review Letters, Vol. 86, No. 2, pp. 5055-5058. 